Review Article

\section{Polyolefin nanocomposites based on metallocene catalysts}

\author{
Walter Kaminsky* \\ Institute for Technical and Macromolecular Chemistry, University of Hamburg, Hamburg, Germany
}

In recent years polyolefin nanocomposites are of great interest because of their high potential as materials with novel properties $[1,2]$. The properties of the nanocomposites are not only influenced by the kind of fillers but also by the microstructure of the polyolefin, the distribution of the fillers, and the preparation process. Nanocomposites prepared by extrusion moulding of mixed polyolefin and nanoparticles show often less stability by agglomeration of the nanoparticles. A better distribution is obtained if the polymerization catalyst is absorbed on the surface of the nanoparticles. After adding an olefin a growing film of the polyolefin is covering every nanoparticle (in situ polymerization).

Metallocene/methylaluminoxane (MAO) catalysts can be used for the in-situ polymerization (Figure 1). The MAO is formed bypartial hydrolysis of trimethylaluminumand consists of units witch form a bulky cluster with molecular weights between 1200 and 2000 [3-5]. As metallocenes are mainly used zirconium complexes. The aromatic rings can be bridged such as for $\left[\left(\mathrm{CH}_{3}\right)_{2} \mathrm{Si}\left(2-\mathrm{CH}_{3} \mathrm{Ind}\right)_{2}\right] \mathrm{ZrCl}_{2}$ producing isotactic polypropylene by propene polymerization, while $\left[\mathrm{CH}_{3} \mathrm{C}(\mathrm{Cp})\right.$ (Flu) $\mathrm{ZrCl}_{2}$ gives syndiotactic polypropylene (Figure 2).<smiles>C[Al](C)O[Al](C)O[Al](C)O[Al](C)C</smiles>

Figure 1: Unit structure of MAO with alternating aluminium oxygen bonds. a. $\mathrm{Cp}_{2} \mathrm{ZrCl}_{2}$

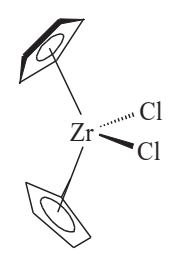

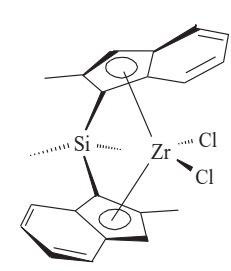

b. $\left[\left(\mathrm{CH}_{3}\right)_{2} \mathrm{C}(\mathrm{Cp})(\mathrm{Flu})\right] \mathrm{ZrCl} 2$

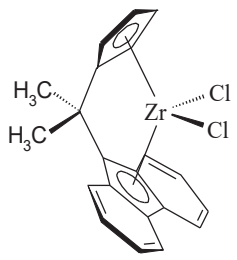

C. $\left[\left(\mathrm{CH}_{3}\right)_{2} \mathrm{C}(\mathrm{Cp})(\mathrm{Flu})\right] \mathrm{ZrCl} 2$

\section{More Information}

*Address for Correspondence:

Walter Kaminsky, Institute for Technical and Macromolecular Chemistry, University of Hamburg, Bundesstr. 45, 20146, Hamburg, Germany,

Email: kaminsky@chemie.uni-hamburg.de

Submitted: January 12, 2021

Approved: February 03, 2021

Published: February 04, 2021

How to cite this article: Kaminsky W. Polyolefin nanocomposites based on metallocene catalysts. Ann Adv Chem. 2021; 5: 001-003.

DOI: 10.29328/journal.aac.1001022

Copyright: (c) 2021 Kaminsky W. This is an open access article distributed under the Creative Commons Attribution License, which permits unrestricted use, distribution, and reproduction in any medium, provided the original work is properly cited.

\section{W) Check for updates}

OPEn Access

Metallocene/(MAO) catalysts are soluble in hydrocarbons and can be easily adsorbed or anchored on the surface of the nanofillers such as particles, fibers, layered silica, carbon nanofiber (CNF), multi-walled carbon nanotubes (MWCNT), changing the surface to a hydrophobic one [6,7]. The MAO reacts, for example, with the $\mathrm{OH}$-groups of silica or with carboxy groups of oxidized carbon nanotubes or is physically absorbed at the surface. Methane is formed by the chemical reaction of MAO with these groups.

Excess MAO is washed out. In a second step, the metallocene is added forming catalytically active polymerization sites on the nanosurface. The thickness of the polymer films, formed by addition of the olefin, depends on the polymerization conditions, especially the polymerization time, the kind of metallocene catalyst, and the pressure of the monomer. Using different metallocenes or olefins a great variety of polymer matrixes can be obtained such as polyethylene, isotactic or syndiotactic polypropylene, ethylene 1-hexene or 1-octene copolymers $[8,9]$. The in-situ polymerization leads to composite materials where the nanoparticles or nanofibers are intensively covered with the polymer.

The composite materials show, for example, an improved stiffness with a negligible loss of impact strength, high gas barrier properties, significant flame retardant, better clarity, and gloss as well as high crystallization rates. Even low nanoparticle contents are already sufficient to obtain new or modified material characteristics, especially a faster crystallization rate and a higher crystallization temperature. 
Carbon nanofibers (CNF) or multiwalled carbon nanotubes (MWCNT) are an especially attractive class of fillers for polymers because of their intriguing mechanical and thermal properties $[10,11]$.

For the preparation the MWCNT were sonicated in a toluene suspension, treated by MAO stirred for 24 hours, filtrated, and washed with hot toluene [12]. After adding the chiral ansa zirconocene $\left[\left(\mathrm{CH}_{3}\right)_{2} \mathrm{Si}\left(2-\mathrm{CH}_{3} \text {-4-Nap-Ind }\right)_{2}\right]$ $\mathrm{ZrCl}_{2}$ (Figure 2) and propene isotactic high molecular weight polypropylene iPP/MWCNT composites with 0.9 - $50 \mathrm{wt} \%$ filler content were obtained. The molecular weights of the polypropylene matrix in the nanocomposites were in the range of $M_{w}=1,200,000-1,700,000$. The polymerization activity reached $5000 \mathrm{~kg} \mathrm{PP} / \mathrm{mol} \mathrm{Zr} \cdot \mathrm{h} \cdot[$ propene]. It was independent of the filler content. As expected for in-situ polymerization, the polymer grew directly on the nanofiber surface and covered them with a thin PP layer. The dried polypropylene nanocomposites were obtained in powder form. By longer polymerization times, the thickness of the polyolefin covering the nanofiber increased. The nanofiber/ $\mathrm{MAO} /$ zirconocene system worked like a supported catalyst. Filler contents between 0.5 up to $50 \mathrm{wt} \%$ were possible.

The morphology of the iPP/MWCNT nanocomposites was investigated by using transmission electron microscopy (TEM). Figure 3 shows the covered nanotubes at two different resolutions, right is the end of a tube by 20 times higher resolution than left.

The diameter of the MWCNT used (about 20 layers) is $20 \mathrm{~nm}$ and the thickness of the iPP coat is about $8 \mathrm{~nm}$. Every nanotube is covered by a polymer film and no agglomeration can be seen. The adhesion of polymer and nanotube is excellent.

The main advantage of CNF or MWCNT filled PP is the change of mechanical properties. High molecular weight isotactic polypropylene filled with MWCNT is an exceptionally strong composite material. The tensile strength of a composite film increases by $20 \%$ if only $1 \mathrm{wt} \%$ of MWCNT is incorporated. Table 1 show the deflection and form stability of these composite materials measured by dynamic mechanical
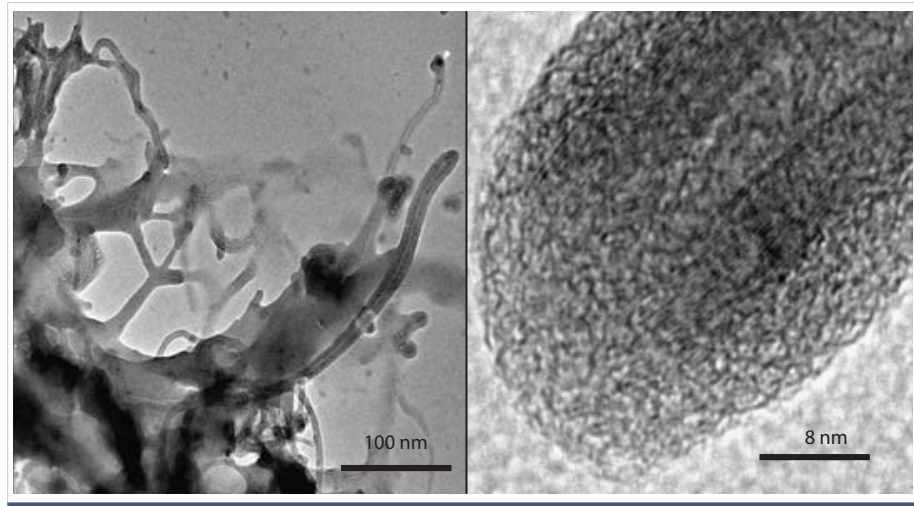

Figure 3: TEM micrographs of a MWCNT composite prepared by in-situ polymerization covered by iPP (Left), top of a nanotube/iPP composite by higher resolution (Right).
Table 1: Deflection and form stability of metallocene based iPP/MWCNT composites, used force: 1.8 MPA.

\begin{tabular}{|c|c|c|}
\hline Filler content $(\mathbf{w t} \%)$ & Deflection at $\mathbf{2 3}{ }^{\circ} \mathbf{C ~} \mathbf{d L}(\boldsymbol{\mu m})$ & Form stability $\mathbf{T}\left({ }^{\circ} \mathbf{C}\right)$ \\
\hline 0 & -488 & 49 \\
\hline 0.9 & -407 & 60 \\
\hline 2.3 & -287 & 71 \\
\hline 3.7 & -231 & 75 \\
\hline
\end{tabular}

analysis. The deflection decreased to the half if the composite contains $3.7 \mathrm{wt} \%$ of MWCNT and the form stability increased by $25{ }^{\circ} \mathrm{C}$. This shows a higher thermal performance of the nanocomposites of nearly $60 \%$. The electrical conductivity starts at a MWCNT content of 5\%. Also the crystallization rate from a melt, important for extrusion moulding processes increase strongly and make this composite material suitable for new applications such as in the automotive plastic industries [13].

Polyolefin nanocomposites open up the approach to new classes of materials with great property combinations. A soft polyolefin matrix can be combined with hard inorganic particles or strong layers of silicates or graphene or with fibers of extreme high tensile strength, such as carbon fibers, carbon nanotubes or polymer fibers. An easy way for the preparation of such polyolefin nanocomposites is the in-situ polymerization using nanoparticles or fibers activated by metallocene/MAO or other single site catalysts. Materials with high gas barrier resistance, high thermal and electrical conductivity, and high form stability can be obtained as well as a good dispersion of the nanofillers in the polymer matrix. The development and commercialization of metallocene/MAO based polyolefin composites have just started and will help in the automotive industries to reduce the weight of a car and by this the energy consumption and is fine for the environmental.

\section{References}

1. Lopez-Gonzalez M, Flores A, Marra F, Ellis G, Gomez-Faton M, et al. Graphene and polyethylene: a strong combination towards multifunctional nanocomposites. Polymers. 2020; 12: 2094.

PubMed: https://pubmed.ncbi.nlm.nih.gov/32942610/

2. McNally T, Poetschke P (eds.). Polymer-Carbon Nanotube Composites: Preparation, Properties, and Applications. Woodhead Publishing, Cambridge, UK. 2011.

3. Kaminsky W. Trends in Polyolefin Chemistry. Macromol Chem Phys. 2008; 209: 459-466.

4. Kaminsky W. Discovery of Methylaluminoxane as Cocatalyst for Olefin Polymerization. Macromolecules. 2012; 45: 3289-3297.

5. Scheirs J, Kaminsky W (eds.). Metallocene-based Polyolefins: Preparation, Properties, and Technology. Wiley, Chichester, UK. 2000; 1,2:

6. Nomura K, Liu KJ. Half-titanocenes for precise olefin polymerization: Effect of ligand substituents and some mechanistic aspects. Dalton Transactions. 2011; 40: 7666-76846.

PubMed: https://pubmed.ncbi.nlm.nih.gov/21409219/

7. Seppälä J, Kokko E, Lehmus P, Malmberg AP, Hakala K, et al Funktional Polyolefins through Polymerization by using Bis(indenyl) zirkonium Catalysts. Adv Polm Sci. 2013; 179-232. 
8. Kaminsky W (Ed) Polyolefins: 50 years after Ziegler and Natta. Vol I and II, Advances in Polymer Science 257 and 258. Springer. Heidelberg. 2013.

9. Alexandre M, Martin E, Dubois P, Marti MG, Jerome R. Polymerization Filling technique: an efficient way to improve the mechanical properties of polyethylene composites. Chem of Materials. 2001; 13: 236-237.

10. Kaminsky W. Metallocene based Polyolefin Nanocomposites. Materials. 2014; 7: 5069-5108.
11. Lozano K, Bonilla-Rios J, Barrera EV. Nanofiber reinforced thermoplastic composites: thermoanalytic and mechanical analysis. J Appl Polym Sci. 2001; 80: 1162-1172.

12. Kaminsky W, Funck A, Klinke C. In-situ Polymerization of Olefins on Nanoparticles or Fibers by Metallocene Catalysts. Top Catal. 2008; 48: 84-90.

13. Kaminsky W. Polyolefin-nanocomposites with special properties by in.situ polymerization. Fronti Chem Sci Engineering. 2018; 12: 555-563. 